\title{
Eficacia de la quinagolida en el tratamiento de un paciente con resistencia hipofisaria a hormonas tiroideas
}

\author{
D. A. DE LUIS*, M. LAHERA, J. I. BOTELLA, M. A. VALERO, C. VARELA \\ *Sec. Endocrinología y Nutrición. Hospital Río Hortega. Instituto Endocrinología y \\ Nutrición. Facultad de Medicina. Valladolid. Departamento Endocrinología. \\ Hospital Ramón y Cajal. Madrid
}

EFFICACY OF QUINAGOLIDE IN A PATIENT WITH PITUITARY RESISTANCE OF THYROID HORMONE

\begin{abstract}
RESUMEN
La resistencia hipofisaria a hormonas tiroideas (RHHT) es una entidad poco frecuente y conocida, su tratamiento sigue siendo tema de controversia. En este trabajo hemos evaluado la eficacia de quinagolida en el tratamiento de una paciente con RHHT. La relación entre tirotropina (TSH) y triyo-dotironina libre (FT3) fue utilizada como marcador de la resistencia tiroidea y de la respuesta al tratamiento. Las concentraciones de TSH y FT3 se normalizaron tras añadir quinagolida al tratamiento antitiroideo clásico con metimazol. Estos resultados sugieren que la quinagolida podría ser un fármaco útil en el tratamiento de esta patología, junto a los tratamientos clásicos.
\end{abstract}

PALABRAS CLAVE: Agentes dopaminérgicos. Hipertiroidismo. Resistencia pituitaria hormonas tiroideas. Quinagolida.

\begin{abstract}
The pituitary resistance to thyroid hormones (PRTH) is not very fre quent and well-known entity, their treatment it continues being topic of controversy. In this work we have evaluated the quinagolida effective ness in the treatment of it unites patient with (PRTH). The relationship among thyroid stimulating hormone (TSH) and free triiodothyronine (FT3) it was used as marker of the thyroid resistance and of the response to the treatment. The concentrations of TSH and FT3 were normalized after adding quinagolida to methimazole. These results suggest that the quinagolida could be an useful drug in the treatment of this pathology, next to the classic treatments.
\end{abstract}

KEY WORDS: Pituitary resistance of thyroid hormone. Hyperthyroi dism. Quinagolide. Dopaminergic agents.

de Luis DA, Lahera M, Botella JI, Valero MA, Varela C. Eficacia de la quinagolida en el tratamiento de un paciente con resistencia hipofisa ria a hormonas tiroideas. An Med Interna (Madrid) 2001; 18: 259-261.

\section{INTRODUCCIÓN}

La resistencia a hormonas tiroideas fue descrita por primera vez por Refetoff $(1,2)$. Posteriormente, un subtipo de resistencia, la resistencia hipofisaria a hormonas tiroideas (RHHT), fue descrita por Gershengorn (3). Este último proceso se asocia a hipertiroidismo debido al exceso de hormonas tiroideas circulantes, presentando los pacientes los síntomas típicos del hipertiroidismo. En estos últimos años, los análisis de DNA han permitido identificar los defectos genéticos en el receptor de hormonas tiroideas que causan este síndrome (4-5).

El tratamiento de los pacientes con esta patología es necesario, sobre todo en los que presentan RHHT, ya que esta suele manifestarse como hipertiroidismo florido, con todas sus consecuencias. Aunque se han utilizado muchos agentes terapéuticos como, la triyodotironina (6), d-tiroxina (7), fármacos antitiroideos clásicos (metimazol, carbimazol) (8), análogos de la somatostatina (9), 3,5,3-ácido triiodothyronineacetic (TRIAC) $(10,12)$, y bromocriptina (11), el tratamiento de elección en esta patología permanece por determinar.
En este caso, demostramos la normalización de TSH y FT3 en una mujer con RHHT, al añadir quinagolida al tratamiento con metimazol. Este tratamiento no había sido utilizado con anterioridad en el tratamiento de esta patología, siendo nuestro caso el primero que ilustra su utilidad en la RHHT.

\section{CASO APORTADO}

Mujer de 20 años de raza caucasiana estudiada en nuestro Servicio por hipertiroidismo y bocio. Los síntomas se iniciaron cuando la paciente tenía 17 años con aumento del tamaño del tiroides, junto a palpitaciones, irritabilidad, pérdida de peso, intolerancia al calor y sudoración profusa. Los análisis de laboratorio mostraron; concentraciones de tiroxina libre (FT4) $3,34 \mathrm{ng} / \mathrm{dl}$ (normal: 0,8 $2,2$ ), triyo-dotironina libre (FT3) $2,46 \mathrm{ng} / \mathrm{dl}$ (normal: $<1,74)$ y TSH $1,89 \mathrm{mU} / \mathrm{L}$ (normal: $0,3-5,5$ ) (fase $0=$ fase de diagnóstico), con anticuerpos antitiroideos negativos. El producto FT3xTSH fue elevado: 4,65 (normal: $2,1 \pm 2,2 \mathrm{ng} / \mathrm{dlx} \mathrm{mU} / \mathrm{L}$ ).

El resto de resultados de laboratorio, tales como una leve hiperglucemia y colesterol bajo eran consistentes con el diagnóstico de hiperti-

Trabajo aceptado: 30 de Septiembre de 1999

Correspondencia: Daniel de Luis Román. c/ Caamaño 51 bis $3^{\circ}$ C. 47013 Valladolid. 
roidismo. Además de las concentraciones elevadas de FT4 y FT3 y normales de TSH, estas últimas aumentaron con la prueba de estimulación con (TRH) (Tabla I). EL resto de determinaciones hormonales fueron normales (Tabla I). La tomografía computarizada y la resonancia magnética cerebrales no mostraron anomalías en el área hipotalámico-hipofisaria. Estos resultados sugieren que el hipertiroidismo no era primario, y en cambio sí cumplía los criterios de RHHT.

\section{TABLA I}

\section{ESTUDIO HORM ONAL HIPO FISARIO}

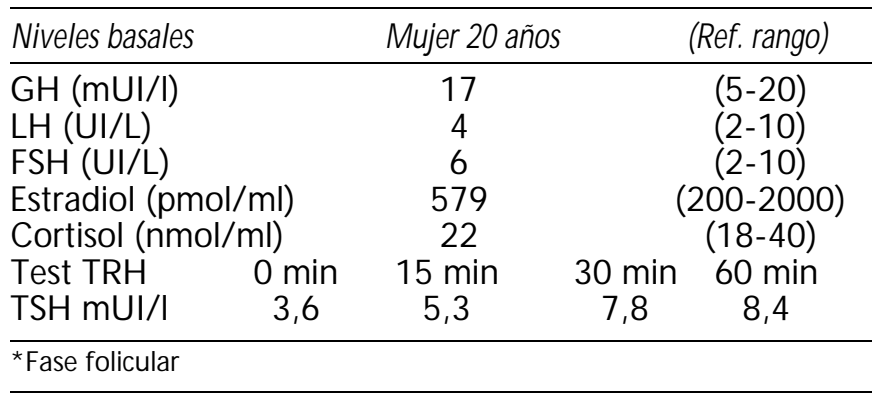

La paciente fue tratada durante dos años con metimazol, primero con $10 \mathrm{mg}$ /día (fase I) y posteriormente con $25 \mathrm{mg} /$ día (fase II). Durante estas fases las concentraciones de FT3 y FT4 no alcanzaron la normalidad (FT3 1,9 ng/dl, FT4 2,4 ng/dl, y TSH 1,57 mUI/ml en la fase I y FT3 1,97 ng/dl, FT4 1,9 ng/dl, y TSh 8 mUI/ml en fase II). Los productos FT3xTSH en estas fases estaban elevados, 2,98 y 15,7 , respectivamente. Después de estas dos primeras fases, añadimos al tratamiento quinagolida, $25 \mathrm{mg} /$ día los primeros días, 50 $\mathrm{mg}$ /día durante 3 días y finalmente $75 \mathrm{mg}$ /día. Con quinagolida las concentraciones de FT3 (1,3 ng/dl), FT4 (1,52 ng/dl) y TSH $(0,32$ $\mathrm{mUI} / \mathrm{ml})$ se normalizaron, así como el producto FT3xTSH $(0,41)$, y desapareció la clínica de hipertiroidismo (fase III), durante 9 meses.

Las concentraciones séricas de FT3 y FT4 se determinaron mediante radioinmunoensayo (Lisophase kits, Laboratory Bouty, Sesto S. Giovanni, Italy). La TSH fue determinada mediante inmunoradioensayo (Auto-DELFIA Wallac, Gaitersburg, Germany). Para estudiar la resistencia a hormonas tiroideas se utilizó el producto FT3 y TSH, este producto se ha mostrado elevado en los pacientes con resistencia, tanto en los que presentan resistencia general como en los que sólo tienen resistencia hipofisaria $(4,6-40,8 \mathrm{ng} / \mathrm{dL} \times \mathrm{mU}-/ \mathrm{l})$, en los individuos normales este producto es de $2,1 \pm 2,2 \mathrm{ng} / \mathrm{dl} \times \mathrm{mU} / \mathrm{L}$ (13). En nuestro paciente, el producto FT3 y TSH se mantuvo elevado en las fases 0 ,I y II, y se normalizó en la fase III.

\section{DISCUSIÓN}

Se han utilizado una gran cantidad de fármacos para tratar la RHHT, pero en pocos de ellos se ha constatado la eficacia del tratamiento a largo plazo.
La triyodotironina se ha demostrado efectiva, aunque su efecto es limitado debido a su corta vida media $(6,10)$. La Dtiroxina también consigue suprimir la TSH y mejora los síntomas y signos de hipertiroidismo en los pacientes con RHHT (7), aunque aún es necesario confirmar su efectividad a largo plazo. El TRIAC también se ha utilizado en esta patología $(9,10)$ y ha sido propuesto como el tratamiento de elección. De cualquier modo, algunos autores han descrito pacientes en los que no se normaliza la TSH y existe un hipermetabolismo continuo (12). Incluso aunque triyodotironina, D-tiroxina y TRIAC disminuyen las concentraciones de TSH, todos estos fármacos presentan una actividad metabólica intrínsica, que hace que, a pesar de normalizar las concentraciones hormonales, los síntomas de hipertiroidismo no desaparezcan.

La somatostatina, un péptico que originalmente se utilizó como inhibidor de GH, también puede disminuir las concentraciones de TSH en los pacientes con resistencia a las hormonas tiroideas. Pero, la somatostatina y sus análogos de larga duración, como el octreotido, no parecen suprimir de manera persistente la TSH, debido a un "fenómeno de escape" tras dos semanas de tratamiento (9); otras desventajas de estos preparados son su alto coste y la necesidad de su administración parenteral.

Es conocido clásicamente que la dopamina disminuye la secreción de TSH. También se ha demostrado que los agentes dopaminérgicos son efectivos en el tratamiento de los cuadros de hipersecreción de TSH debido a adenomas hipofisarios (11) y pueden producir disminución persistente de las concentraciones de TSH en los cuadros de resistencia generalizada a las hormonas tiroideas $(14,15)$ y en la resistencia hipofisaria (16). En algunos casos (17), se ha mostrado que la administración de pergolida, disminuye de manera marcada las concentraciones de TSH y el "pico" de TSH tras TRH. No obstante, en un caso (18), la bromocriptina no pudo disminuir la TSH. En otro caso (11), la TSH disminuyó en el curso del tratamiento con bromocriptina. En nuestro caso, la administración de quinagolida disminuyó las concentraciones de TSH, FT3 y FT4, durante varios meses. La quinagolida es un agonista dopaminérgico no ergotamínico que actúa selectivamente en los receptores D2. Esta molécula ha sido diseñada para que presente los efectos de la bromocriptina sin tener la acción ergotínica, responsable de sus efectos secundarios.

En nuestro caso, se demuestra por primera vez la utilidad de quinagolida para normalizar las concentraciones hormonales a largo plazo en la RHHT. Se han utilizado múltiples fármacos para el tratamiento, algunos de ellos con escaso éxito, por producir muchos efectos secundarios o con "fenómenos de escape". Los nuevos agentes dopaminérgicos, debido a su potencia y sus escasos efectos secundarios, pueden ser utilizados en esta patología.

\section{Bibliografía}

1. Refetoff STL, DeWind LT, DeGroot LJ. Familial syndrome combining deaf mutism, stipled epiphyses, goiter and abnormaly high PBI: Possible target organ refractorines to thyroid hormone. J Clin Endocrinol Metab 1967; 27: 279-294.

2. McKusick VA. Thyroid hormone unresponsiveness (Refetoff syndrome; resistance to thyroid hormone); Mendelian Inheritance in Man. Bat timore, Johns Hopkins University Press 1989; 120-1210.

3. Gershengorn MC, Weintraub BD. Thyrotropin-induced hyperthyroi dism caused by selective pituitary resistance to thyroid hormone. J Clin Invest 1975; 56: 633-642.

4. Sakurai T, Takeda K, Ain K, Ceccarelli P, Nakai A, Seino S, Bell GI, Refetoff S, De Groot LJ. Generalized resistance to thyroid hormone associated with a mutation in the ligand-binding domain of the human thyroid hormone receptor beta. Proc Natl Acad Sci USA 1989; 86: 8977-8981. 
5. Sasaki S, Nakamura H, Tagami T, Miyoshi Y, Tanaka K, Imura H. A point mutation of the $\mathrm{T} 3$ receptor beta 1 gene in a kindred of generalized resistance to thyroid hormone. Mol Cell Endocrinol 1992;84:159-166.

6. Rosler A, Livtin Y, Hage C, Gross J, Cesari E. Familial hyperthyroidism due to inappropiate thyrotropin secretion succesfully treated with triiodothyronine. J Clin Endocrinol Metab 1982; 54: 76-82.

7. Harmon P, Bovier-Lapierre M, Robert M, Peynaud D, Pugeat M, Orgiazzi J. Hyperthyroidism due to selective pituitary resistance to thyroid hormones in a 15-month-old boy: Efficacy of D-thyroxine therapy. J Clin Endocrinol Metab 1988; 67: 1089-1093.

8. Ohzeki T, Egi S, Egawa M, Hachimori K. Thyroid hormone unresponsiveness in two siblings with intrauterine growth retardation and exophtalmos. Eur J Pediatr 1984; 141: 181-183.

9. Beck-Peccoz P, MAriotti S, Guillausseau PJ, Medri G, Pisciteli G, Bertoli A, Barbarino A, Rondetna M, Chanson Ph, Pinchera A, Faglia G. Treatment of hyperthyroidsm due to inappropiate secretion of thyrotropin with the somatostatin analog SMS 201-995. J Clin Endocrinol Metab 1989; 68: 208-214.

10. Salmela PI, Wide L, Juustila H, Ruokonen A. Effects of thyroid hormones (T4,T3), bromocriptine and TRIAC on inappropiate TSH hypersecretion. Clin Endocrinol 1988; 28: 497-507.

11. Takamatsu J, Moza T, Kuma K. Bromocriptine therapy for hyperthyroidism due to increase thyrotropin secretion. J Clin Endocrinol Metab 1984: 58; 934-936.
12. Kunitake JM, Hartman N, Henson LC, Lieberman J, Williams DE, Wong M, Hershman JM. 3,5,3-Triiodothyroacetic acid therapy for thyroid hormone resistance. J Clin Endocrinol Metab 1989; 69: 461466.

13. Ohzeki T, Hanaki K, Motomuzi H, Matsuda Ohtahara H, Ishitani N, Shiraki K. Refractoriness at peripheral and pituitary receptors in general and pituitary types of thyroid hormone resistance. Horm Metab Res 1992; $24: 484-487$

14. Cooper DS, Landenson PW, Nisula BC, Dunn JF, Chapman EM, Ridgway EC. Familial thyroid hormone resistance. Metabolism 1982; 31: 504-509.

15. Bajorunas DR, Rosner W, Kourides IA. Use of bromocriptine in a patient with generalized resistance to thyroid hormone. J Clin Endocrinol Metab 1984; 58: 731-735.

16. Ohzeki T, Hanazi K, Motozumi H, Ishitani N, Urahitani N, Urashima H, Tsukuda T, Shiraki K. Efficacy of bromocriptine administration for selective pituitary resistance to thyroid hormone. Horm Res 1993; 39: 229-234.

17. Sriwatanakul K, McCormic K, Woolf P. Thyrotropin (TSH)-induced hyperthyroidism: Response of TSH to dopamine and its agonists. J Clin Endocrinol Metab 1984; 58: 255-61.

18. Magee B, Sheridan B, Scanlon MF, Atkinson AB. Inappropiate thyrotropin secretion, increased dopaminergic tone and preservation of the diurnal rhythm in serum TSH. Clin Endocrinol 1986; 24: 209-215. 